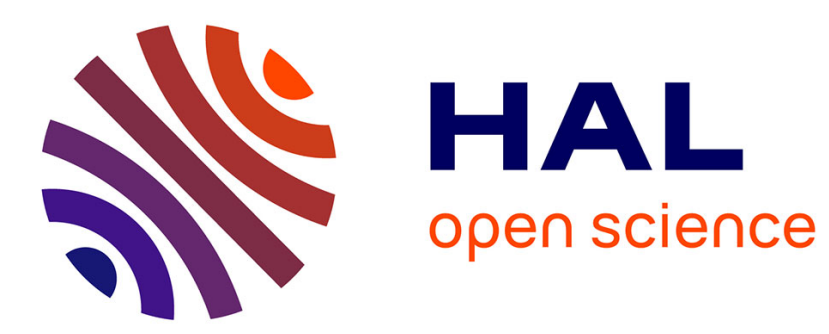

\title{
Idéologie langagière et idéologie tout court : l'exemple de Sorbeval. Roman jurassien de Virgile Rossel \\ Marinette Matthey
}

\section{To cite this version:}

Marinette Matthey. Idéologie langagière et idéologie tout court: l'exemple de Sorbeval. Roman jurassien de Virgile Rossel. Gilles Corminboeuf et Marie-José Béguelin. Du système linguistique aux actions langagières. Mélanges en l'honneur d'Alain Berrendonner, De Boeck Duculot, pp.577-591, 2011, Champs linguistiques, 978-2-8011-1647-0. hal-00956469

\section{HAL Id: hal-00956469 \\ https://hal.science/hal-00956469}

Submitted on 6 Mar 2014

HAL is a multi-disciplinary open access archive for the deposit and dissemination of scientific research documents, whether they are published or not. The documents may come from teaching and research institutions in France or abroad, or from public or private research centers.
L'archive ouverte pluridisciplinaire HAL, est destinée au dépôt et à la diffusion de documents scientifiques de niveau recherche, publiés ou non, émanant des établissements d'enseignement et de recherche français ou étrangers, des laboratoires publics ou privés. 


\section{Idéologie langagière et idéologie tout court : l'exemple de Sorbeval. Roman jurassien de Virgile Rossel ${ }^{1}$}

Marinette Matthey

Univ. Grenoble-Alpes, Lidilem, F-38040 Grenoble

Pour voir clair dans ce qui suit, il faut être averti que aussi bien celui qui écrit ces lignes, que le lecteur qui les lit, sont euxmêmes des sujets, donc des sujets idéologiques (proposition tautologique), c'est-à-dire que l'auteur comme le lecteur de ces lignes vivent «spontanément» ou « naturellement» dans l'idéologie, au sens où nous avons dit que «l'homme est par nature un animal idéologique ». L. Althusser (1970, p. 46).

Dans L'éternel grammairien, Alain Berrendonner propose de considérer le discours normatif comme une idéologie en s'appuyant sur le célèbre texte d'Althusser (1970) Idéologie et appareils idéologiques d'État. Premièrement, le discours normatif, comme l'idéologie en général, vise à modeler les pratiques des individus-sujets auxquels il s'adresse sur le mode de l'interpellation «Eh, vous, là-bas, faites (dites) ceci et pas cela ! ». Deuxièmement, l'idéologie avance masquée derrière de pseudo-constatations. Le discours normatif n'est donc pas ouvertement prescriptif mais plutôt descriptif et il dissimule ainsi ses visées d'assujetissement en fonctionnant sur le mode « il n'y a pas d'alternative, les choses sont comme elles sont, il ne peut en aller autrement ». Troisièmement, le discours idéologique n'a pas d'énonciateur bien défini (mais une majuscule) et les individus se doivent d'entrer dans une relation d'identification (relation spéculaire dit Althusser) avec ce Supersujet immatériel (l'Eglise, les Ancêtres, le Parti, le Bon usage...) qui les fait « marcher tout seuls ».

Le discours normatif aurait pour fonction première selon Berrendonner d'obliger les gens à se classer comme « bons » ou « mauvais locuteurs » (à l'instar des « bons » et des « mauvais sujets » au sein des appareils idéologiques d'état que sont l'Ecole, la Famille, la Justice, les Médias, etc.).

L'idéologie n'est donc pas seulement un système de représentations possédant sa logique propre $^{2}$, mais l'activité même qui consiste à communiquer à d'autres cette architecture qui permet aux sujets interpelés de se positionner. La visée pragmatique modélisante et discriminante du discours normatif est ainsi soulignée (« si vous voulez être quelqu'un de bien ne dites pas ceci mais cela »), de même que ses dimensions axiologiques (Berrendonner $1982: 83$ et ss).

Dans les pages qui suivent, inspirées également par la lecture d'Althusser mais aussi par les écrits sur la notion d'idéologie langagière (Silverstein 1979, Woolard 1998, Kroskrity 2004), j'aimerais montrer comment le discours sur la langue agit comme idéologie tout court dans le roman de Virgile Rossel Sorbeval (1925). Les commentaires du narrateur — que l'on peut

\footnotetext{
${ }^{1}$ Paru dans G. Corminboeuf \& M.-J. Béguelin (sous la direction de) (2011). Du système linguistique aux actions langagières. Mélanges en l'honneur d'Alain Berrendonner (pp 577-992). Bruxelles : DeBoeck Duculot.

${ }^{2}$ Selon la célèbre citation d'Althusser extraite de « Pour Marx » (que je n'ai pas lu mais qui accède au statut de verset biblique grâce à sa reproduction sur des dizaines de sites internet consacrés à l'idéologie) : « Une idéologie est un système (possédant sa logique et sa rigueur propre) de représentations (images, mythes, idées ou concepts selon les cas) doué d'une existence et d'un rôle historiques au sein d'une société donnée. Sans entrer dans le problème des rapports d'une science à son passé (idéologique), disons que l'idéologie, comme système de représentations se distingue de la science en ce que la fonction pratico-sociale l'emporte en elle sur la fonction théorique (ou fonction de connaissance) $»$.
} 
sans peine assimiler à l'auteur lui-même — sur les façons de s'exprimer de ses personnages et la manière dont il les fait parler se prêtent à une telle analyse. Je traiterai ces extraits comme des représentations linguistiques plus ou moins explicitées, qui renvoient à une idéologie de la langue française à la fois comme objet sacré (comme le dit l'instituteur Léon Dufresne

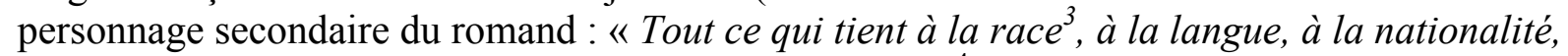
à la religion, rentre dans les catégories sacrées. ", p. 94) 4 et chargée d'une mission civilisatrice. Par idéologie langagière, il faut entendre des croyances rationalisées à propos de la forme et de l'usage de la langue, en lien avec l'identité, l'esthétique et la morale, et qui s'appliquent par métonymie au groupe social (à la « race ») qui parle cette langue.

Après avoir brièvement présenté l'auteur et le roman, je traiterai successivement de trois figures (ou ensembles de figures) convoquées dans et construites par le récit : le bon et le mauvais étranger d'une part, les indigènes de l'autre.

\section{Qui est Virgile Rossel?}

L'auteur est né à Tramelan en 1858. Docteur en droit, il sera avocat avant d'être appelé en 1884 par Joseph Stockmar (membre jurassien du gouvernement bernois) pour occuper une chaire extraordinaire de droit français à l'Université de Berne. Il sera recteur en 1893 et 1907. Membre du parti radical, il est élu conseiller national en 1896. Juge fédéral de 1912 à 1932, il s'installe à Lausanne où il meurt en 1933. Il est un des coauteurs du Code civil suisse de 1917. Parallèlement à sa carrière professionnelle et politique, il écrit des poèmes, des romans, des pièces de théâtre, une biographie d'Eugène Rambert (1830-1886, lui-même biographe et poète suisse) et une autre sur Louis Ruchonnet (1834-1893, figure du radicalisme vaudois), ainsi qu'une Histoire de la littérature française hors de France (1895), un des premiers écrits sur la « francophonie » qui s'inscrit dans le sillage d'Onésime Reclus (1837-1916) et de son célèbre France, Algérie et colonies (1886). Virgile Rossel a 67 ans lorqu'il publie Sorbeval.

\section{Sorbeval. Roman jurassien}

Sorbeval parait à Lausanne en 1925. C'est un roman à thèses puisque la préface de l'auteur thématise explicitement le conflit jurassien et que l'histoire contée n'est finalement qu'un prétexte pour faire passer un message politique, celui de la lutte contre la « germanisation du Jura ». Ce message s'accompagne toutefois d'un appel aux valeurs suisses de la négociation et de la franchise : " Me blâmera-t-on d'avoir appelé, ou rappelé, l'attention sur un problème qu'il serait préférable d'ignorer? Ce n'est pas en se taisant qu'on fera que ce qui est ne soit pas. Rien de plus efficace, rien de plus digne, rien de plus suisse en un mot, que le recours à la franchise pour dissiper équivoques et malentendus. » Le message politique est clairement entendu par les lecteurs et les militants, bien des années après la première édition du roman. André Muller rappelle ainsi « l'opinion autorisée d'un de nos plus hauts magistrats » dans un article rédigé en 1947 pour la brochure Comment on germanise le Jura et Sorvebal est réédité en 1987 par le Groupement « Sorbeval» de Tramelan, qui avertit le lecteur dans un avantpropos : « Qui veut comprendre la Question jurassienne doit lire « Sorbeval » car Virgile Rossel a vécu le drame jurassien dans son cœur et dans sa chair. [...]. En un réquisitoire courageux, vibrant, vigoureux, il lance un véritable cri d'alarme » (p. 7). La menace, c'est la germanisation du Jura. En 1987 le canton du Jura existe depuis 9 ans, mais la patrie est

\footnotetext{
3 Au début du vingtième siècle, le terme « race » n'a pas encore les connotations négatives qu'on lui connait aujourd'hui. C'est un synonyme de « peuple » ou d'« ethnie ». On le trouve encore dans cette acception en 1954 sous la plume d'Alfred Lombard (recteur de l'Université de Neuchâtel entre 1925 er 1927) : « On n'oublie pas que la Suisse est la réunion de terres vivant de leur vie propre, de terres qui ont façonné une race et dont la voix s'exprime dans un parler natal ». Cahiers de l'Institut neuchâtelois No 4, p. 26.

${ }^{4}$ La pagination correspond à la réédition de Sorbeval par le Groupement « Sorbeval » de Tramelan, 1987. Les extraits du roman sont cités entre guillemets et en italiques.
} 
toujours déchirée puisque le sud est resté dans le canton de Berne, à majorité germanophone. Le groupement « Sorvebal » continue donc la lutte (« La libération du Jura méridionnal est inéluctable », p. 8) et en appelle désormais au droit international pour légitimer la revendication séparatiste: « toutes les minorités ethniques, linguistiques et religieuses ont droit au respect de leur culture, de leur religion et de leur langue. » (p. 8).

Précisons enfin que Sorbeval est un village qui n'existe pas, et que ce n'est donc pas un roman à clé. Mais comme le dit l'auteur dans sa préface : «J'ai observé, je me suis souvenu, et l'imagination a fait le reste ». Le lecteur familier du territoire imaginera sans peine une vallée du Jura sud et ne peut s'empêcher de traduire Gros-Mont par Chasseral, la Raisse par la Suze et Seeblick par Bienne. Quant à l'auteur, il était lui-même fils du maire de Tramelan, à l'instar de Julien, un des personnages secondaires de son roman, "frêle adolescent si peu fait pour les rudes travaux de la campagne ou le milieu malsain de l'atelier » (p. 58), qui fréquente l'Ecole cantonale de Porrentruy (comme Rossel), passe son temps libre à lire Rousseau sous la douce férule de son oncle et fera son droit pour ouvrir une étude d'avocatnotaire à Sorbeval.

\section{Les figures du roman : le bon étranger, le mauvais et les indigènes}

La manière dont Virgile Rossel fait parler les protagonistes et/ou les commentaires du narrateur à propos de leur manière de s'exprimer permettent de reconstruire trois catégories de personnages qui dessinent les contours de l'idéologie de l'auteur dans ses dimensions exo et endogroupales. L'opposition entre le « bon » vs le « mauvais étranger» est centrale. A ces représentations de l'exogroupe des Bernois germanophones s'ajoutent celles de l'endogroupe des francophones jurassiens, sous la forme de quelques commentaires sur le français parlé à Sorbeval qui illustrent l'ambivalence des Suisses romands face au français régional, à la fois symbole d'une identité propre et stigmate de la périphérie (De Pietro \& Matthey 1993).

\section{Le bon étranger}

Fritz Emmenried, domestique bernois, est le principal représentant de cette figure. Rossel nous le montre dès la première page du roman remontant les gorges de la Raisse et interpelant un habitant du coin dans un "français approximatif que n'allégeait point l'accent bernois : combien jusqu'à Sorbeval ? (...) Il s'obstinait, et pour cause, à prononcer : Sorpefal » (p. 11 et 12). Mais il parle déjà français, langue qu'il a apprise durant la Première guerre mondiale, alors qu'il était mobilisé dans le Jura nord, et il ne demande qu'à s'améliorer en sollicitant « humblement » des leçons auprès de Juliane, la fille de Daniel Desforges, son patron. Leçons qu'elle ne lui accorde pas toujours (elle a parfois la migraine) mais «Emmenried se contentait de ce qu'on lui offrait ou de ce qu'on ne lui refusait pas, et qui valait mieux que rien. Pourvu qu'il trouvât plus facilement ses mots et qu'il perdit son terrible accent... ». (p. 31). Les leçons portent leurs fruits et la jeune fille peut même explorer avec Fritz «l'un des maquis de notre orthographe : les participes passés » (p. 88).

Le premier extrait est un des passages clés du roman, d'ailleurs repris par Müller dans Comment on germanise le Jura sous le titre « La responsabilité du pasteur allemand ». Il met en scène la rencontre de Fritz Emmenried et du pasteur Gottlob Karlen, deux Bernois germanophones de Sorbeval. L'enjeu du choix de langue révèle les options acculturatives bien différentes du « bon » étranger qui désire s'assimiler et du « mauvais » qui se croit en territoire conquis.

Extrait 1 (p. 32-33)

Le monsieur à la vertigineuse allure jette à Emmenried un « Grüss Gott» haletant ; un tranquille « bonsoir » lui répond qui, malgré les leçons de Juliane, est de Trub infiniment plus que de Sorbeval. Aussitôt de montrer au domestique du maire un visage désséché et rougeaud, sillonné à gauche par de profondes balafres, et d'apostropher Emmenried en sonore dialecte d'Outre-Thièle. 
- De quelle commune es-tu?

- Je suis de Trub

— « Je suis de Trub ». Auriez-vous honte de parler l'allemand?

Il a posé sa seconde question en français. Tout naturellement, comme il passe d'un idiome à l'autre, il substitue le : vous au : tu.

- Ce n'est pas la langue d'ici.

- Drôle de raison! Depuis quand êtes-vous dans le Jura?

- Depuis le 15 juin.

- Je ne vous ai pas vu à l'église, cet après-midi.

- J'ai assisté, ce matin, au sermon de M. Perrelet.

- C'est moi qui suis votre pasteur, le pasteur Gottlob Karlen.

- Ah!

— Le gouvernement s'intéresse à ceux des nôtres qui habitent le Jura. Chaque quinzaine j'ai un culte à Sorbeval. Je compte que maintenant...

— Je regrette M. Perrelet prêche en français [...] puisque je suis chez les welches, je veux vivre avec les welsches et comme eux.

Les balafres que Karlen avait rapportées de l'Université de Tübingue se creusèrent et passèrent au rouge lie de vin [...] Reprenant son patois et tutoyant Emmenried, Karlen riposta :

— [...] il y a trois commandements essentiels pour toi : ne pas renier ta langue maternelle ; ensuite...

— Ça doit être bien égal au bon Dieu qu'on le prie en allemand ou en français.

- ... ensuite, être fier de ta race ; enfin, avoir des principes religieux... Nous nous reverrons.

Ce dialogue entièrement en français (mis à part la salutation initiale) parvient tout de même à faire imaginer la scène en suisse-allemand et en français tout en montrant la dynamique des choix de langues et leurs répercussions immédiates sur la catégorisation des locuteurs. Aux yeux de Karlen, Emmenried est un traitre... mais son discours d'assimilé fait de lui un bon Bernois aux yeux des habitants de Sorbeval, et de Rossel lui-même. L'utilisation du vouvoiement coïncide avec le passage au français du pasteur, ce qui montre, outre la bonne capacité d'observation sociolinguistique de Rossel, sa croyance en un degré de civilité supérieur incorporé au français: cette langue permet de mettre de la distance et du respect urbain entre les individus alors que le dialecte ne connait que la fraternité informelle et rustique. Nous sommes en plein dans l'idéologie portée par Onésime Reclus, qui voit dans le français « l'idiome supérieur, digne de sa réputation de langage le plus vif et le plus civilisé de l'Europe $»^{5}$. Face au domestique paisible qui a adopté la langue de civilisation, le pasteur parle le dialecte sonore d'Outre-Thielle et porte des balafres qui exhibent le caractère belliqueux et agressif des Germains ${ }^{6}$. La sonorité de la langue, le discours comminatoire et le visage du pasteur créent une isotopie de la laideur, de la raideur et du mal qui souligne à contrario à quel point Emmenried est dans le vrai, le juste et le bon, mais aussi combien l'harmonieuse et raisonnée civilisation française est plus désirable que l'agressive et bornée civilisation allemande.

Emmenried, on s'en doute, finira par épouser la fille de son patron, suite à une laborieuse déclaration d'amour en français, enfin osée à la fin du roman par le domestique suisseallemand "garçon sans éducation, sans instruction et qui n'a pas d'autre bien que ses deux bras » (p. 117). De plus, " il est d'une autre race, il n'a pas cette finesse, cette souplesse, ce liant, cette générosité insoucieuse, ce désir et ce besoin de plaire qu'ont les welches » (ibid.), mais malgré cela, Juliane lui fait l'immense honneur de le prendre pour époux et le père Desforges ne s'y oppose pas :

\footnotetext{
5 France, Algérie et colonies, p. 413.

${ }^{6}$ La raison de ces balafres pourrait être militaire. Il était semble-t-il « de bonne tradition chez les officiers allemands au XIXe siècle d'avoir des balafres au visage, souvent acquises dans des duels à l'épée. Elles étaient une marque de courage au combat et de fierté ». Pierre Bühler, Université de Zurich, communication personnelle.
} 


\section{Extrait 2 (p. 119)}

— Puisque vous êtes d'accord, mariez-vous [...] Juliane sera Mme Emmenried... Emmenried-Desforges ! Pour être franc, si j’avais été Juliane, la question du nom et de la race... Les femmes sont les femmes ! [...] Le diable, mon bon Fritz, c'est que tu viennes de l'autre bout des gorges... On te welschisera... Toujours est-il qu'en voici un de plus à Sorbeval ! [...] Vous avez de l'appétit... Après tout, si vous nous mangiez, vous ne nous digéreriez pas.

Le dernier tableau du roman nous montre Emmenried, désormais maitre du domaine, houspillant un domestique avec juste « un soupçon d'accent bernois dans [son] français ». (p. 124). Ses enfants, Fritz et Daniel, ne parlent évidemment que cette langue (cela va tellement de soi que Rossel ne nous le dit même pas), Emmenried est parfaitement assimilé. Les races se sont mélangées pour le meilleur ${ }^{7}$ et il est devenu jurassien francophone, comme Péter Messerli, un autre migrant alémanique de Sorvebal qui laisse de temps en temps échapper « un mot désagréable pour des oreilles jurassiennes » (p. 35) lorqu'il perd aux cartes (le lecteur est prié d'inférer : un juron en suisse-allemand), mais ce bref changement de langue involontaire ne saurait lui être reproché. Le mélange des races, symbolisé par les prénoms des deux enfants, n'est pas stigmatisé, mais il s'opère sous le couvert de l'assimilation linguistique au français. Là encore, on retrouve une idée chère à Onésime Reclus : les races peuvent se mélanger, pour autant que les individus-sujets se retrouvent au sein d'une même langue : " dès qu'une langue a " coagulé » un peuple, tous les éléments « raciaux » de ce peuple se subordonnent à cette langue $[. .$.$] la langue fait peuple. { }^{8}$ Le roman s'achève sur un dernier dialogue entre Daniel Desforges, père de la mariée et maire de Sorbeval, et l'oncle Adam-Louis (l'adorateur de Rousseau) à propos de son désormais beau-fils :

Extrait 3 (p. 123)

— «(..) Emmenried serait, un de ces quatre matins, candidat du parti paysan au Grand Conseil que ça ne me surprendrait pas du tout... Respect pour mon gendre ! Je n'étais pas très fier du choix de Juliane. Un domestique, un Allemand !... Mais Emmenried a le nez creux et la bosse des affaires (...) Un peu braque, le garçon, et il nous conduirait tous à la baguette s'il n'y avait pas Juliane. Non pas qu'elle soit un dragon en tablier de cuisine. Elle a sa façon à elle de le prendre.

- C'est que Juliane...

- Est une Desforges, parbleu!

Le mélange des races a été fécond : le couple a engendré deux garçons et une fille (donc trois Jurassiens), Fritz Emmenried a épongé les dettes de son beau-père grâce à son sens aigu des affaires, et sa sobriété toute germanique aura eu de l'effet sur le Jurassien un peu trop porté sur les petits verres de goutte au café : Daniel Desforges a signé la tempérance 9 . Virgile Rossel mobilise deux stéréotypes bien connus des Romands et des Alémaniques pour camper ces personnages : les premiers sont « légers » (léchères, comme le prononcent souvent les Romands, imitant l'accent de leur compatriotes) et les seconds «profitieren » ( $\mathrm{p}$. 17), c'est-à-dire qu'ils « profitent toujours de l'occasion ${ }^{10}$ », qu'ils ont un sens aigu de la conduite des affaires. Mais ces stéréotypes sont très ambivalents : la légèreté est condamnable quand elle pousse à s'adonner à la boisson, mais positive lorsqu'il s'agit de style langagier et

\footnotetext{
${ }^{7}$ Près de trente ans plus tard, Marcel Godet (dans « La langue française ») considère également que l'immigration des Alémaniques dans le canton de Neuchâtel « constitue à certains égards un apport utile comme élément de rajeunissement ethnique ». Cahiers de l'Institut neuchâtelois, 1954, p. 13)

${ }^{8}$ Un grand destin commence, p. 116.

9 « Temperance, pop. Tempé, s. f. Engagement d'abstinence des boissons alcooliques : signer la tempérance (ou simplementt signer) ». William Pierrehumert, Dictionnaire du parler neuchâtelois et suisse romand, 1926.

${ }^{10}$ Le Dictionnaire Suisse romand relève un emploi régional profiter de + inf. ou en emploi absolu avec le sens de « profiter de l'occasion » et précise qu'on le rencontre fréquemment en Suisse alémanique dans la langue de la publicité (« Profitieren Sie »).
} 
d'éducation. Le côté " profitieren » est condamnable lorsqu'il conduit à s'approprier le bien d'autrui sans accepter de devenir comme lui (comme on va le voir avec la figure du mauvais étranger), mais digne d'admiration lorsqu'il relève du sérieux, du travail et du sens des affaires. Rossel fait preuve d'une pondération et d'un sens du compromis jusque dans ses stéréotypes, révélant par là sa nature profondément suisse (et radicale)!

\section{Le mauvais étranger}

Face à cette figure du « bon » étranger qui contribue à la vitalité de la population jurassienne, se dresse celle de Hans-Uli Zürcher, fermier anabaptiste de la Chevalette (qu'il s'obstine à nommer Rossboden, au grand dam du propriétaire Daniel Desforges). L'extrait suivant présente la scène où le fermier vient faire une offre d'achat du domaine dont il est seulement locataire, mais qu'il a bien l'intention d'acquérir en " profitant» du malheur du paysanmaire : ce dernier a été ruiné par un affairiste bernois qu'il avait eu la mauvaise idée de cautionner, et il s'est en plus cassé la jambe en pourchassant le voleur Albiser. Il reçoit son fermier couché dans son lit, sans pouvoir se lever. Il est bien dans une position de faiblesse et Hans-Uli va en profiter...

\section{Extrait 4 (p. 81-82)}

\footnotetext{
- Bonjour, Grüss Gott. Neige tombe pas, cette année. Tombera . Sentir rhumatismes.

L'anabaptiste Hans-Uli Zürcher, le fermier de la Chevalette, usait d'un français bizarre, tout en ellipses, tout farci d'allemand bernois, et que Daniel appelait le « négro-tütch ». [...]

— Commissions à Sorbeval... Profite pour régler compte avec vous.

- Un peu d'argent ne sera pas de refus.

De la gorge de Hans-Uli s'échappa une sorte de gazouilli moqueur.

— Hé, hé... Ha, ha !... Hi, hi !... Vous devoir Geld à moi.

- Elle est mauvaise !

— Moi prouver. Beaucoup réparations... Très chers, ouvriers, très chers. Und...

- Und quoi ?

Dans son charabia, Hans-Uli exposa laborieusement [toutes les dépenses qu'il avait dû engager] - Ai chagrin pour vous, monsieur maire. Benggeli, Lump, canaille. Fabricant de Seeblick, cautionné par vous, au-dessous de ses affaires. Et votre Unfall... Albiser, aussi une canaille. A volé cinq poules à moi, avant-hier...
}

Hans-Uli parle une variété de français présentée que l'auteur, via son personnage jurassien, nomme « négro-tütsch ». Le sens de l'observation sociolinguistique de Rossel est cette fois moins pointu : la variété qu'il met dans la bouche du Bernois est assez peu vraisemblable. Certains traits sont typiques de la Variété de Base décrite par Klein et Perdue (1997) (absence de flexion verbale, verbe implicite, absence de détermination...), mais Hans-Uli emploie tout de même le futur et surtout un lexique un peu trop élaboré pour une interlangue ( Fabricant de Seeblick cautionné par vous »). Ce français « bizarre et farci d'allemand bernois » (p. 81) sert à épingler le mauvais migrant, celui qui vient coloniser la terre jurassienne, refuse d'abandonner sa langue rustique pour le français et surtout n'hésite pas à changer les toponymes ancestraux ( La Chevalette, pour nous de la montagne, plus la Chevalette: Rossboden »).

Le fantasme de n'être plus maitre chez soi s'exprime dans une maxime mise dans la bouche du pasteur Perrelet : "Vivre et laisser vivre ceux chez lesquels on vit » (p. 92), phrase qui de l'avis de l'oncle rousseauiste est sublime : «il n'y en avait pas une, dans les CEuvres complètes de Rousseau, qui fut d'une plus sobre, ni d'une plus saisissante éloquence. » (ibid.). Le fantasme d'être « dévorés » par l'envahisseur bernois (même si mal « digérés », cf. extrait 2) se nourrit d'une activité angoissante de classification des patronymes des votants de la commune : "En consultant les registres électoraux, Dufresnes découvrit que, sur trois cents quarante-six noms, cent soixante-deux étaient à désinence germanique. » (p. 91). La toponymie et l'onomastique se voient investies d'enjeux symboliques déterminants, qui 
expliquent les légères réticences du père à l'idée du mariage de sa fille : ce sont moins les considérations sociales (un domestique qui épouse la fille du maire) que symboliques qui les entrainent (sa fille change de nom, reçoit un nom germanique qu'elle transmettra à ses descendants, même si Juliane aura à coeur de faire suivre son nom d'épouse par celui de son père, cf. extrait 2 ).

Le mauvais étranger est donc celui qui veut faire sienne la terre du Jura mais qui ne veut pas renoncer à la langue de sa « race », l'allemand de Berne, à laquelle il n'a pourtant plus droit puisqu'il est chez les welsches (cf. extrait 1). Il patique aussi l'endogamie ${ }^{11}$. En bref, le mauvais étranger refuse de se plier à l'injonction assimilatrice du peuple jurassien, qui est aussi une injonction civilisatrice de la Langue française (on ne dit pas encore Francophonie). Le Jura est en effet une « petite France hors de France ${ }^{12}$ et les migrants alémaniques, à l'instar des indigènes d'Onésime Reclus, ont tout intérêt à abandonner leur dialecte (alémanique pour les premiers, arabe pour les seconds) au profit d'une langue qui a infiniment plus de valeur universelle grâce à son potentiel civilisationnel harmonieux. En effet, comme le note Provenzano (2010 : 97), l'enjeu de la francophonie selon Reclus est bien d'opposer une colonisation heureuse et irénique face à celle perçue comme uniquement agressive et conflictuelle de l'Allemagne (symbolisées par les balafres du pasteur Karlen, comme on l'a vu ci-dessus, extrait 1). L'impression dérangeante que les Bernois sont un peu à Rossel ce que les Algériens sont à Reclus est renforcée par la dénomination dévalorisante de négro-tütch, qui fait irrésistiblement penser à celle de " petit-nègre », expression qui fleure bon l'époque des colonies, et qui est définie, selon les dictionnaires, comme un français rudimentaire, incorrect, parlé par les indigènes des colonies françaises (Amedegnato \& Sramski 2003 : 16). Si en Algérie le colonisation française a pour mission de civiliser les indigènes, dans le Jura, c'est l'inverse : les « colons bernois » seront civilisés par la langue et la culture française de ses habitants ! Dans les deux cas, il s'agit bien de s'assimiler à cette grande langue de civilisation pour s'incorporer à la « francophonie » (désignation anachronique, mais c'est bien cette idée de francophonie universelle qui est en train d'apparaitre).

Face aux autres et à leur manière de parler et d'apprendre ou de ne pas apprendre le français, il y a donc les Jurassiens. Comment Rossel envisage-t-il leur facon de parler ?

\section{Les indigènes}

Le français parlé par les Jurassiens du roman est très standard. Mais Rossel n'hésite pas à mimer l'oral de manière plaisante (dislocations, co-énonciation, registre familier) ni à utiliser des régionalismes en les signalant par des guillemets. Ils sont la plupart du temps mis dans la bouche de Daniel Desforges : «je peux bien me « $\operatorname{corder}^{13}$ » une once de bon sang 》 (p. 30), «c'est que j'ai un " gouvernement » plus raide que la justice de Berne » (p. 17) (pour parler de sa femme). Dans la description d'une activité typique comme le jeu de cartes, Rossel utilise les expressions locales, avec un brin de distance condescendante quand même : «ils étaient trop passionnément attentifs à leur jeu pour échanger entre eux autre chose que des

\footnotetext{
${ }^{11}$ Cette question n'est pas explicitement thématisée dans le roman, mais l'auteur parle bien des « familles anabaptistes », germanophones, on peut déduire qu'elles sont endogames.

12 On trouve cette formule dans l'introdution à l'ouvage Histoire de littérature française hors de France, également rédigée par le prolixe Rossel : «Cette langue a été l'instrument d'une vie intellectuelle ardente et riche à ses heures, et c'est parfois un sentiment d'admiration étonnée qu'on éprouve devant le travail accompli, selon l'esprit et au profit du génie de leur race, par ces trois petites France hors de France [i.e. La Belgique, le Canada et la Suisse romande] ». (p. 1)

13 « S'accorder, se payer », origine dialectale (francoprovençal) mais qui correspond aussi à l'allemand gönnen (Pierrehumbert).
} 
bouts de phrases baroques et peu variés : «j'ai les nelle »; - "stoeck »- ; «les bours ${ }^{14}$; " atout, atout, atout » " (p. 35). Daniel Desforges ne craint pas non plus les jurons comme «Tonnerre des Indes » (que l'on trouve dans La Maison à vapeur de Jules Verne et qui n'est pas une expression locale, non plus que «tu peux te brosser le ventre » (p. 18), mis dans la bouche d'un voisin de Desforges). On ne trouve par ailleurs que très peu de formes locales sans guillemets (je n'en ai débusqué que trois), et on ne peut pas savoir si Rossel les utilise sciemment. L'absence de guillemets pourrait nous laisser penser que non. Il s'agit du fameux question tag typique de la Suisse romande « ou bien » ("Hé c'est encore le père qui commande. Ou bien? »), d'un «billon ${ }^{15} »$ et d'un «lundi bleu » ${ }^{16}$ qui apparait dans un dialogue symbolisant bien la relation entre le père paysan et le fils futur " gratte-papier », qui a de la peine parfois à se lever le matin :

Extrait 5 (p. 42)

- On n'aurait pas eu besoin de t'arracher de ton lit, si tu n'étais pas resté jusqu'à minuit chez ton oncle à lire des bêtises.

- Nous nous sommes oubliés dans l'Emile de Rousseau, un livre...

- Oh ! un livre comme les autres. Tu ferais mieux de te coucher en même temps que nous. Tu as une figure de lundi bleu.

Mis à part le maire Desforges, qui semble moins sensible au prestige des lettres que l'auteur du roman, les Jurassiens sont présentés comme des gens bien éduqués, y compris les femmes, ce qui est progressiste pour l'époque, notons-le (Rossel est aussi l'auteur d'un roman taxé par lui-même de «féministe » ${ }^{17}$ !) :

La future épouse de Fritz, Juliane, a fait une école en Suisse allemande: "Après une année et demie de pensionnat à Waedenswyl, dans le canton de Zurich pour apprendre l'allemand, elle était rentrée à Sorbeval aussi simple de goûts et aussi peu ménagère des ses bras qu'à l'heure $d u$ départ. » (p. 19). On a vu plus haut à quel point Fritz se sent honoré d'êre remarqué par cette jeune femme à la fois instruite, modeste et travailleuse (des valeurs portées par le radicalisme protestant de Rossel) ; Julien, le frère de Juliane, fait donc des études, lit l'Emile, et sa constitution fragile lui interdit les travaux des champs ou de l'usine et l'oncle AdamLouis Mallet, vieux sage qui consacre sa vie à Rousseau, a transmis sa passion à son neveu... Il faut encore ajouter à cette liste de personnage l'instituteur du village, qui épousera une jeune fille ayant accompli un apprentissage et exerçant le métier de relieuse (encore les livres...).

Si les Jurassiens sont des gens instruits, ils n'en sont pas moins de la périphérie, mais ils l'assument. Une scène du roman brosse la rencontre entre deux « sociétés de jeunesse » au sommet du Gros-Mont : celle de Sorbeval (Jura sud) et celle de la Chaux (Jura nord). Cette scène donne l'occasion à Rossel de renvoyer tous les Jurassiens dos-à-dos (extrait 6) et de stigmatiser légèrement la facilité d'un calembours lancé par un Jurassien du nord qui a un peu forcé sur le vin blanc de Neuchâtel (extrait 7) :

Extrait 6 (p. 48)

— Qu'est-ce que ces particuliers-là ? grommèle le fils Käser. Des Montagnons, sans doute, à en juger par leur accent.

\footnotetext{
${ }^{14}$ Désination du neuf et du valet d'atout dans le jeu de cartes appelé Jass ou stöck, très populaire en Suisse romande : cf. Pierrehumbert STEUQUER, FAIRE UN STEUC.

15 « Bille, tronc, pièce de bois en grume ou écorcée provenant du sectionnement d'un tronc («plante ») et destinée à être sciée en planche ». Ce sens est commun à la Suisse romande (Pierrehumbert).

16 « Jours de loisirs supplémentaires que s'accordaient quelques ouvriers horlogers ». Répertoire officiel des plaques explicatives de la toponymie urbaine de la Chaux-de-Fonds (PASSAGE DES LUNDIS-BLEUS).

${ }^{17}$ Ce que femme veut..., roman féministe, Neuchâtel, 1931.
} 


\section{Extrait 7 (p. 52)}

Et c'est le moment du départ ! La fanfare de la Chaux peut ne pas quitter le Gros-Mont avant la nuit : elle n'a pas « charge de dames », comme le rappelle Jacques Frésard, non sans tirer quelque vanité de ce médiocre à-peuprès.

Périphérie ne signifie pas pour autant insignifiance ou rejet des racines locales. Au contraire, Rossel fait dire à un Jurassien du sud qui a les larmes aux yeux en écoutant ceux du nord chanter les Vâlats de Mieco (Les valets de Miécourt) qu'il est bien dommage que "dans le Val des Sorbes, le patois ne soit plus guère qu'un souvenir » (p. 49). Daniel Desforges cite un proverbe en patois: "La grand'maman Desforges avait raison : "Après lai risatte, lai pueratte », - après les rires, les pleurs » (p. 81). Nulle trace dans le roman d'un sentiment de honte envers ce patois, ce qui contraste assez fortement avec la valeur négative du terme en France, qui lui vaut d'ailleurs d'être encore aujourd'hui farouchement combattu par les linguistes de l'Hexagone. Rossel voit le patois comme la langue des ancêtres garante de l'originalité et de l'indigénat du peuple jurassien. Il ne menace pas le français mais le complète.

Il est plaisant de constater que la Constitution jurassienne de 1978 contient un article sur la défense du patois et que le canton s'est doté en octobre 2010 d'une loi concernant l'usage de la langue française, dont l'article 9 alinéa a) stipule : " L'Etat assure un enseignement qui permet la maitrise et suscite l'amour de la langue française ». Voilà qui doit combler d'aise les mânes de Virgile Rossel !

\section{Conclusion}

Des quelques analyses qui précèdent, il me semble pouvoir tirer les deux conclusions suivantes à propos de l'idéologie de Virgile Rossel:

- Le combat jurassien basé sur l'idée de la Rauracie une et indivisible va de pair avec une conception essentialiste des peuples. Essentialiste mais non raciste puisque Rossel valorise au contraire l'assimilation et le mélange des « races » sous la bannière du français.

- Au-delà du plaidoyer pour l'assimilation linguistique des migrants germanophones, Virgile Rossel est un des premiers diffuseurs de l'idéologie « francodoxe » (Provenzano 2011), basée sur des croyances en la supériorité naturelle de la langue française, dont la France est le « foyer central».

La francodoxie perdure aujourd'hui, mais elle a changé de discours. La proclamation des valeurs de la démocratie et des droits de l'homme a remplacé le discours sur les progrès de la civilisation, mais la francophonie s'invente toujours une vocation universelle liée à une langue et à des valeurs qu'elles porteraient spécialement bien en raison de son histoire. « Le français revendique sa vertu rassembleuse, donnons-lui sens et substance en nous inspirant de la diversité culturelle, de l'amitié et de la fraternité entre les peuples ». Ce n'est pas Virgile Rossel qui le dit, mais Elisabeth Baume-Schneider, ministre jurassienne et présidente de la CIIP en $2011^{18}$. Les idéologies langagières ne se démodent pas facilement !

\footnotetext{
18 Editorial du programme de la $16^{\mathrm{e}}$ semaine de la langue française et de la francophonie, Conférence intercantonale de l'Instruction publique des cantons romands et du Tessin, Neuchâtel, 2011.
} 


\section{Textes utilisés (par ordre chronologique)}

Onésime Reclus. France, Algérie et colonies. Paris, Hachette, 1887 [en ligne] http://gallica.bnf.fr/ark:/12148/bpt6k75061t

Virgile Rossel. Histoire de la littérature française hors de France. Lausanne, F. Payot, 1895.

Onésime Reclus : Un grand destin commence. Paris, La Renaissance du livre, 1917 [en ligne] http://gallica.bnf.fr/ark:/12148/bpt6k72918f

Virgile Rossel, Sorbeval. Roman jurassien. Delémont, Imprimerie jurassienne S.A., 1991 [1925].

Jean Gressoz, Roland Béguelin, André Muller, Roger Chatelain \& André Schwander, Comment on germanise le Jura. Porrentruy et Tramelan, Cahier spécial de la « Cité Nouvelle », 1947.

Marcel Godet, Alfred Lombard, Eddy Bauer, René Braichet, Eric Berthoud, Georges Redard, La langue française. Entretiens de Neuchâtel. Cahiers de l'Institut neuchâtelois No 4, 1954. Neuchâtel, Editions de la Baconnière.

\section{Bibliographie}

Althusser, L. (1970). Idéologie et appareils idéologiques d'État. (Notes pour une recherche). Version électronique établie par Jean-Marie Tremblay [Article originalement publié dans La Pensée no 151, juin 1970, puis dans Positions (1964-1975). Paris : Les Éditions sociales, 1976, pp. 67-125. [en ligne] http://dx.doi.org/doi:10.1522/030140239.

Amedegnato, S. \& Sramski, S. (2003). Parlez-vous petit nègre? Enquête sur une expression épilinguistique. Pars : L'Harmattan.

Berrendonner, A. (1982). L'éternel grammairien. Berne : Lang

De Pietro, J.-F. \& Matthey, M. (1993). « Comme Suisses romands on emploie déjà tellement de germanismes sans s'en rendre compte... ». Entre insécurité et identité linguistiques: le cas du français à Neuchâtel (Suisse). Cahiers de l'institut de linguistique de Louvain 19. 3-4, 121-136.

Pierrehumbert, W. (1926). Dictionnaire du parler neuchâtelois et suisse romand. Neuchâtel : Editions Attinger.

Provenzano, F. (2011). Vie et mort de la francophonie. Une politique française de la langue et de la littérature. Liège : Les Impression nouvelles (Réflexions faites).

Klein, W. \& Perdue, C. (1997). The Basic Variety. Second Language Research,13 (4), 301347.

Kroskrity, P.V. (2004). Language Ideologies. In : A. Duranti (ed.) A Companion to Linguistic Anthropology (pp. 496-517). Malden, Oxford, etc. : Blackwell Publishing.

Silverstein, M. (1979). Language Structure and Linguistic Ideology. In P. R. Clyne, W. F. Hancks \& C. L. Hofbauer (éds.), The Elements: A Parasession on Linguistic Units and Levels. Chicago: Chicago Linguistic Society, 193-247.

Thibault, A. (1997). Dictionnaire suisse romand. Particularités lexicales du français contemporain. Lausanne : Editions Zoé (sous la direction de P. Knecht et avec la collaboration de Gisèle Boeri et Simone Quenet). 
Woolard, K. A. (1998). Language Ideology as a Field of Inquiry. In B. B. Schieffelin, K. A. Woolard \& P. V. Kroskrity (éds.), Language Ideologies. Practice and Theory. Oxford \& New York: Oxford University Press, 3-47. 